\title{
Role of Ultrasonography and Multi-detector CT in Imaging of Anterior Abdominal Wall Lesions
}

\author{
El-Sayed El-Mekkawy El-Sayed ${ }^{1}$, Tarek Fawzy Abd-Ella ${ }^{1}$, Asmaa Saied Zaian² \\ ${ }^{1}$ Radiology Department, Faculty of Medicine, Menoufia University, Shibeen El Koom, Egypt \\ ${ }^{2}$ Radiology Department, Shebin El-Koom Teaching Hospital, Menoufia, Shibeen El Koom, Egypt
}

Email address:

sayedmekkawy49@yahoo.com (S. M. El-Sayed), Tarek Fawzy@yahoo.com (T. F. Abd-Ella), Said_asmaa80@yahoo.com (A. S. Zaian)

\section{To cite this article:}

El-Sayed El-Mekkawy El-Sayed, Tarek Fawzy Abd- Ella, Asmaa Saied Zaian. Role of Ultrasonography and Multi-detector CT in Imaging of Anterior Abdominal Wall Lesions. International Journal of Medical Imaging. Vol. 3, No. 5, 2015, pp. 98-104.

doi: $10.11648 /$ j.ijmi.20150305.13

\begin{abstract}
This study aim to evaluate the role of US and CT when needed in diagnosis of anterior abdominal wall lesions. Ultrasound and Multi-detector computed tomography are currently the study of choice to diagnose abdominal wall lesions; mostly hernia it also allows accurate identification of abdominal wall hernias and their content, and it allows to assess the possible complications, differentiate from other abdominal masses like: tumors, hematoma, abscess and it helps in the detection of pre- or postoperative complications. This prospective study included 50 patients suffering from abdominal wall swelling, US examination of abdomen was performed for all patients and only 10 patients had under gone US and CT examination the abdomen. The ratio of females to males was 28 to 22 . Their ages ranged from $22-60$ years with mean age of 40 years. Ventral hernia was the most common cause of anterior abdominal wall lesions 26 cases (52\%), anterior abdominal lump 14 cases (28\%), 10 cases were needed to CT evaluation. Ventral hernia was the most common lesions (30\%) 3 cases out of 10 cases, strangulated hernias (20\%) 2 cases, spegilan hernia, anterior abdominal wall abscess and desmoid tumor (10\%) 1 case for each. US is the prime investigation for patients suspected of having abdominal wall swellings and was the first step in the diagnosis while CT scan was a sensitive tool for imaging and we recommend it as a confirmative imaging method.
\end{abstract}

Keywords: CT, US, Hyper-Echoic, Hypo-Echoic, Hyper-Dense

\section{Introduction}

Abdominal wall lesions often mimic intra-abdominal conditions and frequently present as palpable masses this is more common with patients who have a thick abdominal wall with a large layer of fat. (1)

There are wide range of pathology affecting the anterior abdominal wall which range from simple fluid collection to hernias to complex neoplasms of the abdominal wall hence early detection of these pathology with use of high resolution USG and other cross sectional imaging have revolutionized the treatment options for the surgeons. (2)

Anterior abdominal wall hernias are the most common lesion of anterior abdominal lesions (2)

With the introduction of high-frequency, high-resolution probes, detailed examination and recognition of different layers of the abdominal wall are now possible on USG examinations. A high-resolution examination is capable of deciding whether an abnormality is in the abdominal wall or inside the abdominal cavity. Physical findings in abdominal wall pathologies have low specificity and often a clinically suspected intra-abdominal lump proves to be in the abdominal wall (3)

US gives its lack of ionizing radiation and lower cost, and may be used as a first-line imaging modality in certain radiation-sensitive populations (e.g., pediatric and pregnant patients) or in patients with suspected subcutaneous masses because of its high sensitivity and specificity. (3)

CT imaging, which is relatively more costly and involves ionizing radiation, but the use of CT can result in savings in time for diagnosis and overall cost of hospitalization and may then be reserved for cases requiring further problem solving secondary to indeterminate US findings or for detecting lesions not visible on US due to body habits and or overlying bowel gas. (3)

The aim of this work is to highlight the role of US and 
$\mathrm{CT}$ in the diagnosis of anterior abdominal wall lesions.

\section{Material and Methods}

\subsection{Patients}

This prospective study included 50 patients with abdominal wall swelling and abdominal pain referred from surgical department in Shibeen-el Kom teaching hospital. They included 22 males and 28 females with age distribution 22- 65 years with mean age of 41 years. These patients were referred to abdominal ultrasound and 10 cases only were referred to US and MDCT. All patients underwent careful history intake, full clinical examination and surgical confirmation.

\subsection{Ultrasonography}

Scanning the abdominal wall requires no special patient preparation. The examination can be performed over surgical wounds by applying an adhesive plastic membrane over the wound after removing the dressing; the adhesive is sterile and prevents both contamination of the wound by the transducer by an infected wound or draining sinus. The patient should lie comfortably on his /her back (supine). The head may rest on a small pillow and if there is much abdominal tenderness, a pillow may also be placed under the patient's knees. The abdomen is covered with coupling agent. The patient can be allowed to breathe quietly, a real time survey of the entire abdominal wall is performed with superficial probe (7.5-10) $\mathrm{MHz}$ transducer and with convex deep probe (3- 5) $\mathrm{MHz}$ transducer for some obese cases then scanning in transverse scan then longitudinal scan. The morphological features including swelling location and its relation to the surrounding structures, it's content, echogenicity and vascularity of swelling were evaluated.

\subsection{Computerized Tomography}

CT scan of the abdomen was done after explanation of the procedure and it's contraindications to the patients. All patients were examined by 4 detectors $\mathrm{CT}$ scanner. On anterior-posterior scout view, the area of interest was indicated. Images were acquired from starting the diaphragm to the symphysis pubis were taken in full inspiration, axial cut sections were taken with a thickness of $10 \mathrm{~mm}$ contiguous sections, $3 \mathrm{~mm}$ when examining small organs (pancreas, adrenal glands ), during a single breath-hold, with the patient supine, the arms were raised and placed behind the patient's head, out of the scan plane. Feet first on the scanner table, with the median sagittal plane perpendicular to the table, field of view (FOV) is ranged from $15-50 \mathrm{~cm}$ according to thickness of abdomen, Matrix: 256, Radiation factors: $\mathrm{kV}$ : $120-140$ \& m amp: 200, Window - level: soft tissue window 250-50 - liver window 160-60, patients with suspected anterior abdominal wall lesions would also be imaged while erect. As a substitute for examination in the erect position, we perform CT examinations in the left or right lateral decubitus position, while increasing intra abdominal pressure, by having patients perform a Valsalva's maneuver.

\subsection{Statistical Analysis}

The data collected were tabulated and analyzed by SPSS (Statistical Package for the Social Science software) statistical package version 11 on IBM compatible computer.

Qualitative data are expressed as numbers and percentages (No \& \%).

\section{Results}

Fifty patients presenting with abdominal wall swelling and abdominal pain represent the study group of this thesis, showed a high prevalence of anterior abdominal wall lesions in the age group of $>40 \mathrm{yrs}(54)$, a higher incidence in females i.e. as many as 28 cases (56. \%), abdominal swelling was the most common symptoms of suspected anterior abdominal wall lesions represent 25 cases ( $50 \%$ ), abdominal swelling and abdominal pain represent 22 cases of 50 cases $(44 \%)$ and abdominal swelling and fever represent 3 cases ( $6 \%$ ), ventral hernia was the most common cause anterior abdominal wall lesions as 26 cases ( $52 \%$ ) followed by strangulated hernias i.e. 5 case $(10 \%)$. We would like to emphasize another anterior abdominal wall lesions other than hernias which were common in our study was anterior abdominal wall hematoma i.e. as many as 3 cases $(6 \%)$, anterior abdominal wall abscess represent 3 cases as $(6 \%)$, anterior abdominal wall lipomas and anterior abdominal wall cysts 2 cases $(4 \%)$, and other lesions in our study were desmoids tumor, endometriosis, metastatic nodule and seroma i.e. a case for each lesion ( $2 \%$ ). (Table 1$)$, all hernia cases were clearly diagnosed by US which showed the hernia defect and it's contents as contained (bowel loops only 20 cases out of 36 patients, or omentum only 6 cases $(16 \%)$, or both 10 cases $(30 \%)$. (Table 2$), 10$ cases which needed CT evaluation.

Table No.1. Showing various high resolution sonography of 50 patients of anterior abdominal wall lesions.

\begin{tabular}{lll}
\hline Pathological finding & N & \% \\
\hline Umbilical hernia & 11 & 22.00 \\
Para-umbilical hernia & 11 & 22.00 \\
Epigastric hernia & 4 & 8.00 \\
Incisional hernia & 4 & 8.00 \\
Spigelian hernia & 1 & 2.00 \\
Strangulated hernia & 5 & 10.00 \\
Anterior abdominal wall cyst & 2 & 4.00 \\
Lipoma & 2 & 4.00 \\
Hematoma & 3 & 6.00 \\
Desmoid tumor & 1 & 2.00 \\
Metastic nodule & 1 & 2.00 \\
Endometriosis & 1 & 2.00 \\
Abscess & 3 & 6.00 \\
Seroma & 1 & 2.00 \\
Total & 50 & 100.00 \\
\hline
\end{tabular}




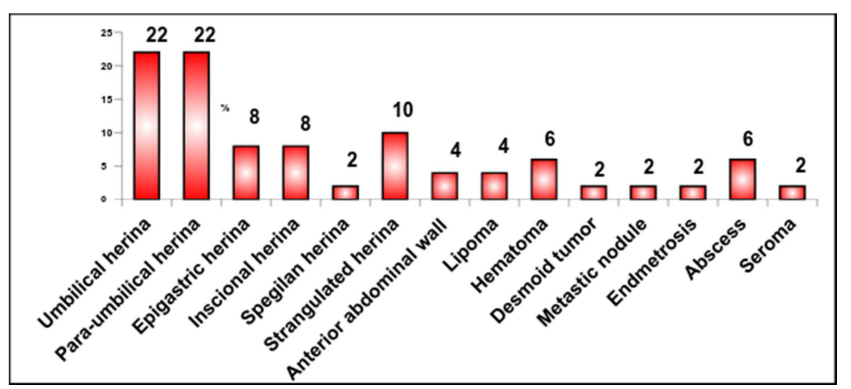

Chart 1. Showing various high resolution sonography of anterior abdominal wall lesions.

Ventral hernia was the most commonly lesions of anterior abdominal wall 3 cases out of 10 cases $(30 \%)$ followed by strangulated hernias i.e. 2 cases $(20 \%)$, another anterior abdominal wall lesions other than hernias which were common in our study, were anterior abdominal wall hematoma, abdominal wall abscess, desmoids tumor and anterior abdominal wall endometriosis i.e. each represented 1 case of 10 cases ( $10 \%)$. (Table 3 )

Table No.2. Shows ultrasound finding in different types of anterior abdominal wall hernia.

\begin{tabular}{lll}
\hline Ultrasound finding & NO. patient & $\mathbf{\%}$ \\
\hline Defect containing bowel loops only & 20 & $56 \%$ \\
$\begin{array}{l}\text { Defect containing bowel loops and } \\
\text { omentum }\end{array}$ & 10 & $30 \%$ \\
Defect containing omentum 0nly & 6 & $16 \%$ \\
Total & 36 & $100 \%$ \\
\hline
\end{tabular}

Chart 2. Showing ultrasound finding in different types of anterior abdominal wall hernia.

Table No.3. Shows 10 patients of anterior abdominal wall lesions were undergone both US and CT study.

\begin{tabular}{lll}
\hline CT findings & & \\
\hline disorders & N & \% \\
\hline Umbilical hernia & 2 & 20.00 \\
Para-umbilical hernia & 1 & 10.00 \\
Spigelian hernia & 1 & 10.00 \\
Strangulated hernia & 2 & 20.00 \\
Abscess & 1 & 10.00 \\
Desmoid tumor & 1 & 10.00 \\
\hline
\end{tabular}
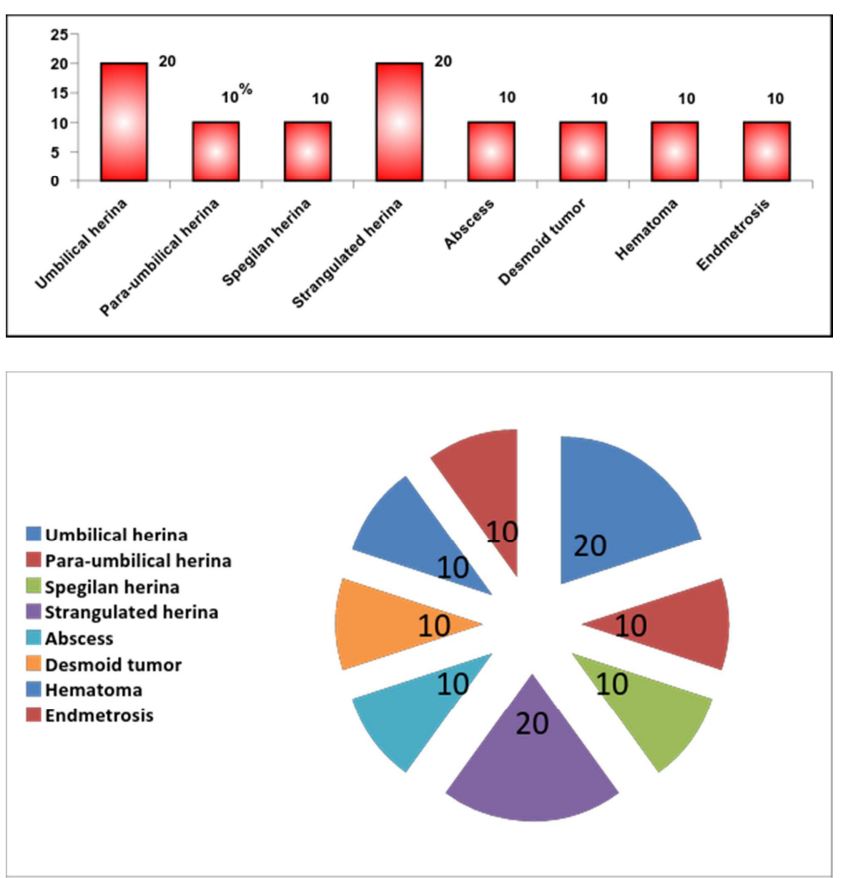

Chart 3. Showing of CT finding of 10 cases of anterior abdominal wall lesions.

\section{Discussion}

There are wide range of pathology affecting the anterior abdominal wall which range from simple fluid collection to hernias to complex neoplasms of the abdominal wall, abdominal wall hernias are a common imaging finding in the abdomen., hence early detection of these pathology with use of high resolution USG and other cross sectional imaging has revolutionized the treatment options for the surgeons.

Our study showed a high prevalence of anterior abdominal wall lesions in the age group of $>40$ yrs. The majority of cases were various types of ventral and incisional hernias 26 cases out of 50 cases, with a higher incidence in females i.e. as many as 28 cases. This is in agreement with (RodriguezHermosa JI, et al),( Halm JA et al), the umbilical hernia is a common surgical problem mainly encountered in the 5th and 6 th decades of life. The umbilical defect is observed in more than $90 \%$ of elderly patients. It is seen mainly in obese patients, especially in women, in patients with liver cirrhosis $(1,5,6)$. The mean age of the patients was 57.1 years, and $68 \%$ of all patients with umbilical hernia were women. $(4,5)$. In most series, women have a higher incidence of ventral hernia than do men but this may be related to the fact that incisional hernia is more frequently seen after hysterectomy, caesarean section and appendicectomy.

4 patients were diagnosed as incisional hernia, (Halm JA,et al) stated that incisional hernia conducted in AlKadhimia teaching hospital in Baghdad and Tikrit teaching hospital during the period from October 2002 to July 2006, in which 75 patients with incisional hernia were assessed, their ages ranged from 6-80 years with a mean age of $36 \pm 4$ $\mathrm{SD}$, forty one females $(54.7 \%)$ and 34 males $(45.3 \%)$. It was found that incisional hernias were common in age group (41- 
50) years among female patients but the incidence was more common among the age group of (41-60) in male patients. The most common site of previous incision was lower midline $(22.7 \%)(5)$

1 patient was diagnosed as spigelian hernia in our study (Louring-Andersen $\mathrm{M}$, et al) stated that, retrospective review of Spigelian hernia patients, twentyfive patients (64.1\%) were females and 14 patients (35.9\%) were males. The mean age was 70 years (range 37-92 years). Left side was the most frequent location, 17 patients (44\%) and three patients $(8 \%)$ had a bilateral Spigelian hernia. Risk factors were present in $74 \%$ of patients and a history of previous abdominal operation was the most frequent one. In $41 \%$ the Spigelian hernia appeared concomitantly with other type of abdominal wall hernia. (7,8), 3 patients were diagnosed as anterior abdominal wall hematoma in our study, (Neblett WW 3rd, et al) stated that 126 anterior abdominal wall hematoma, most patients $(64 \%)$ were women and the mean $+/-$ SD age was $67.9+/-16.5$ years. Most patients (69\%) were on some form of anticoagulation therapy. (9), 3 patients were diagnosed as anterior abdominal wall abscess in our study, (Mura A,et al ) stated that 53 reviewed cases of abdominal wall abscesses in the Japanese literature and reported just three (6\%) spontaneous abdominal wall abscesses. Thirtyone cases (61\%) were due to malignant diseases, followed by inflammatory diseases such as acute appendicitis. The present patient had a previous history of rectal cancer although there was no evidence of recurrent disease found on CT scan, colonoscopy, or at laparotomy (10)

Other lesions in our study were desmoids tumor, endometriosis, metastatic nodule and seroma i.e. A case for each lesion, (Lefevre $\mathrm{JH}$, et al ) stated that 32 desmoid tumor cases a family history of desmoid tumors was found in four patients, all of whom had abdominal wall desmoid tumors. Associated familial adenomatous polyposis (FAP) was found in one patient. Among the 22 abdominal wall desmoids, 19 were present in reproductive aged women (15-45 years), of whom 16 were multiparous with one or more children each. There was a history of abdominal surgery in six patients (27\%), of these five had undergone a LSCS and one had an appendectomy. (11), 1 patient was diagnosed as endometriosis in our study, (Women DOUGLAS C, et al) stated that total of 17 endometriosis patients with anatomical pathological diagnosis of an endometriosis node in a gynecological scar were found. The following variables were studied: the age at diagnosis (32.5 years $+/-5.5$ years), personal and obstetric history, time from surgery to diagnosis ( 4.2 years $+/-3.4$ years), node size $(2.5 \mathrm{~cm}+/-1.1 \mathrm{~cm})$ and location (caesarean scar, $82 \%$; and laparoscopic surgery port, $5.8 \%$ ), 1 patient was diagnosed as metastatic nodule in our study, (GandolfoN, et al) stated that 17 patients presented with nodules in the surgical scar, either single ( $\mathrm{n}=9$ patients) or multiple ( $\mathrm{n}=2$ patients), in 11 patients operated on for known abdominal neoplasm confirmed by histology. The most common primary tumor was colonic carcinoma. Ultrasonography was performed in all cases; contrastenhanced CT was carried out in 10 patients, lesions had variable size $(15-55 \mathrm{~mm})$, roundish shape, ill-defined margins (60\% cases) and hypo-echoic solid echo-texture. All were characterized by marked contrast medium uptake on CT examination. (13), among 50 various clinical suspected anterior abdominal wall lesions which were subjected for color Doppler sonography, the most accurately detected lesions of anterior abdominal wall i.e 30 cases of 50 cases were positive findings, and 20 cases of 50 cases were negative finding $(40 \%)$ as with the application of Color Doppler sonography differentiation between strangulated and non strangulated hernias can be made out. Diagnostic of other anterior abdominal wall lesions as abscess (preserved peripheral vascularity) which represented 3 cases.

In our study 5 patients were diagnosed as strangulated hernia (Stabile Ianora AA, et al ), stated that the frequency of Occurrence of absence of blood flow within hernia as Criterion of hernia incarceration in 126 non incarcerated and 23 Incarcerated Hernias. Data suggest that the absence of blood flow in the contents of a hernia should not be taken as a sign of incarceration because most incarcerated hernias in these series (78\%) had detectable blood flow on color Doppler sonography.

The patients in whom color Doppler sonography did not detect blood flow in the incarcerated bowel loop included the 5 patients with incarcerated hernia and bowel necrosis.(22\%) and 31 from 126 non incarcerated (25\%). (14), 10 patients in our study were needed to CT evaluation. 3 patients were diagnosed as ventral hernia was the most common lesions of anterior abdominal wall followed by strangulated hernias 2 cases, which can detected herinal sac and its contents bowel loops or omental fat and can detected obstructed bowel or not, we would like to emphasize another anterior abdominal wall lesions other than hernias which was common in our study, were anterior abdominal wall hematoma, abdominal wall abscess, desmoids tumor and anterior abdominal wall endometriosis i.e. each represented a case of 10 cases (Stabile Ianora AA, et al ) stated that concerning the value of $\mathrm{CT}$ in diagnosis of abdominal wall hernia of examined 55 patients with suspicion of abdominal wall hernia with ultrasonography thirteen of patients had CT scan performed as well. Finding on CT correlate with sonographic feature, forty - seven patients were diagnosed as abdominal wall hernia but only 12 patients were diagnosed based on surgical finding and sensitivity of findings were 0.83 in CT evaluation and specificity 0.67 . (14)

\section{Conclusion}

Anterior abdominal wall hernias are the most common lesion of anterior abdominal lesions. US is really helpful in patients with inconclusive or misleading clinical presentations. US is non-invasive, allows for comparison with the asymptomatic side and can be performed in physiological positions with dynamic scanning; for these reasons it plays a fundamental role in evaluating the presence of complications such as strangulation or incarceration and, in some cases, US may detect further pathology in the hernial 
sac. CT provides an accurate and panoramic view of the abdomen including more identification of hernias and their contents and differentiation of hernias from other abdominal masses (tumors, hematomas, abscesses). Furthermore, because of its superior anatomic detail, multi - detector row CT may help detect subtle signs of complication within the hernia sac, including bowel obstruction, incarceration, strangulation and traumatic wall hernia.

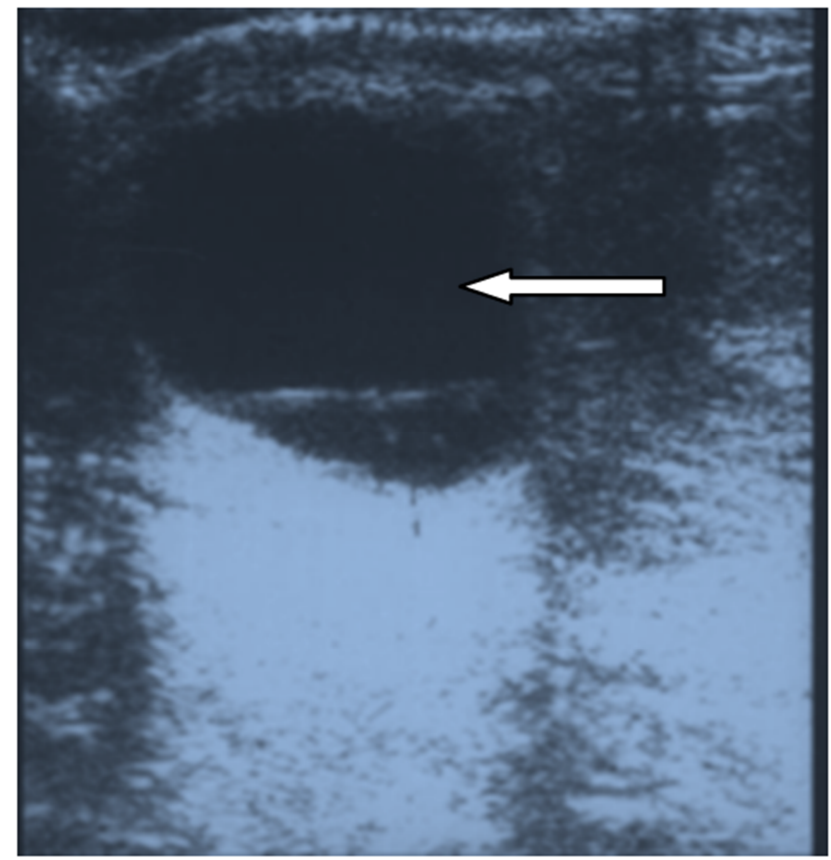

Figure 1. Male patient 25 years old. presented with supra-para-umblical painful swelling since 5 days with fever, Transverse superficial sonography demonstrate Subcutaneous supra- para-umblical well defined Cystic lesions measuring about $(4 \times 5 \mathrm{~cm})$, with thickened wall, fluid debris leveling and posterior acquastic enhancment. (Supra-Para umbilical abscess)

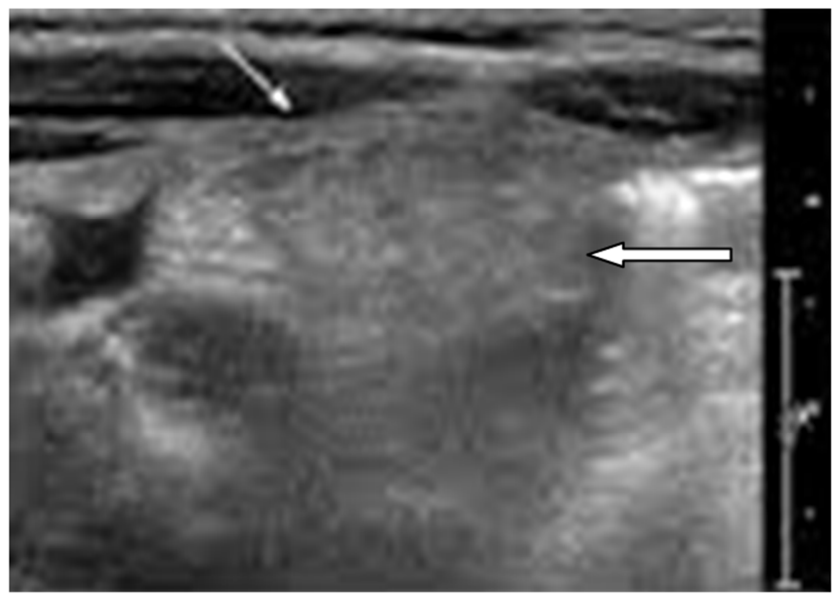

(A)

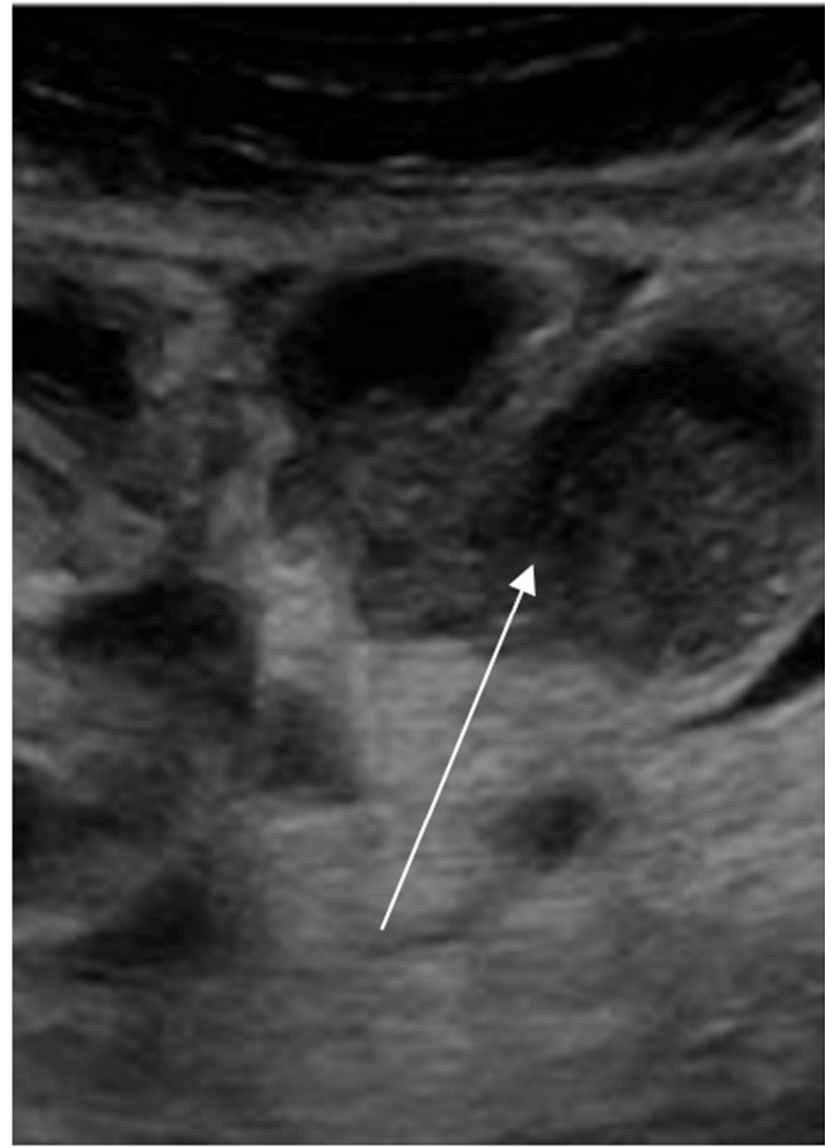

(B)

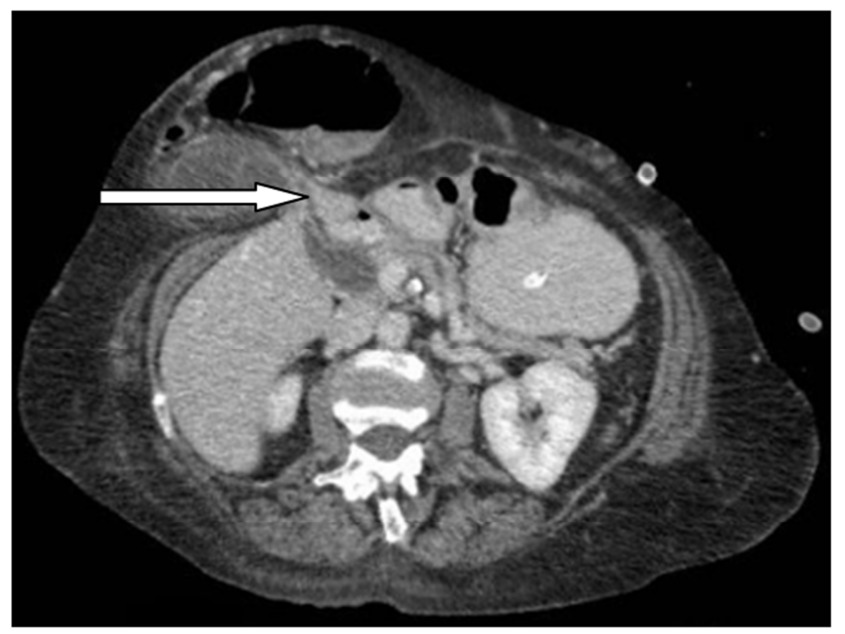

(C)

Figure 2. Male patient 50 years old suffering from supra- paraumblical swelling since 2 months increasing with postural changes and cough with abdominal pain,no fever.Fig.2(A):Transverse US image (superficial probe), demonstrate supra-para-umblical hernia defect in linea alba measuring about $1.7 \mathrm{~cm}$ with sac containing viable bowel loops Fig.2 (B) : Transverse sonography ( by deep probe), demonstrate supra -para umblical hernia defect in linea alba measuring about $1.7 \mathrm{~cm}$ with sac containing viable bowel loops Fig.2 (C) : Axial CT abdomen, demonstrate herination of bowel loops through neck of hernia sac (supra-para umbilical herina) 


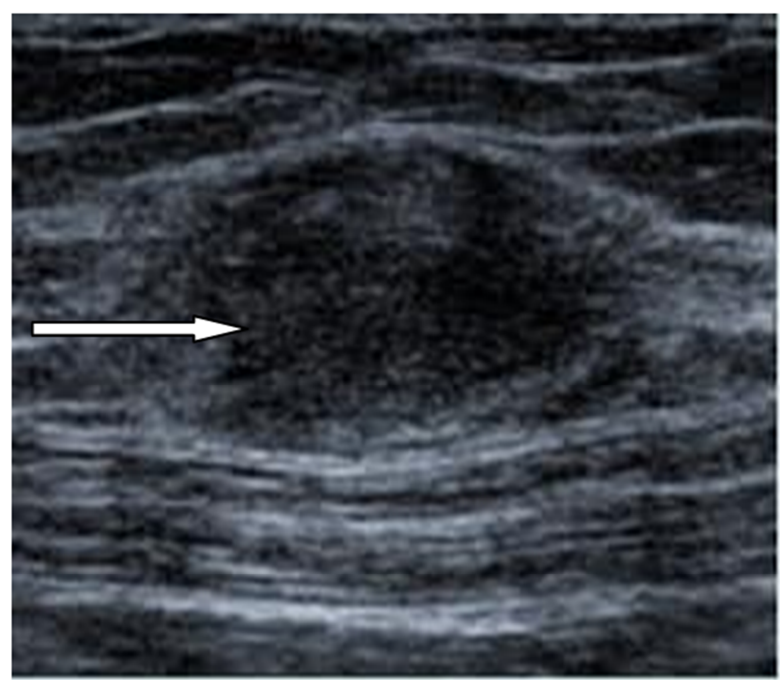

(A)

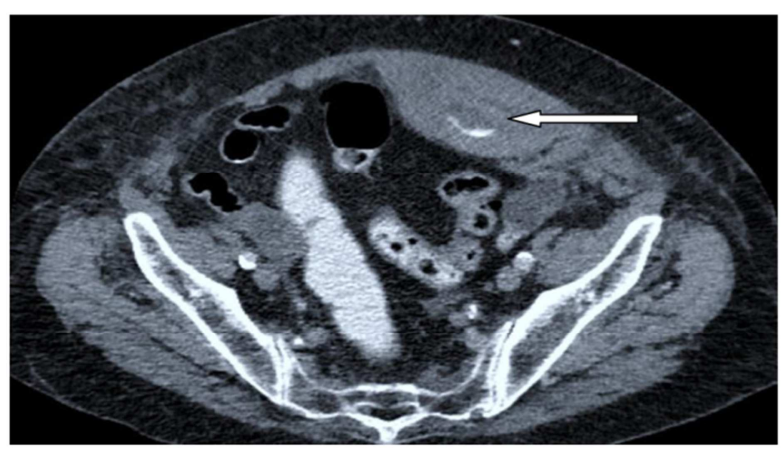

(B)

Figure 3. Male patient 19 years old, presented with paniful abdominal wall swelling at right para-median above umbilicus level after car accident. Fig. 3 (A) :Transverse superficial ultrasonography, demonstrate well defined, regular intramuscular hypoechoic lesion in rectus abdominus muscle measuring about $2 \times 2 \mathrm{~cm}$ (rectus abdominus hematoma) Fig. 3 (B):Axial CT abdomen demonstrate well defined heterogeneous soft tissue density mass lesion at $L T$ rectus abdominus muscle reveling hematoma measuring about $2 \times 2 \mathrm{~cm}$.

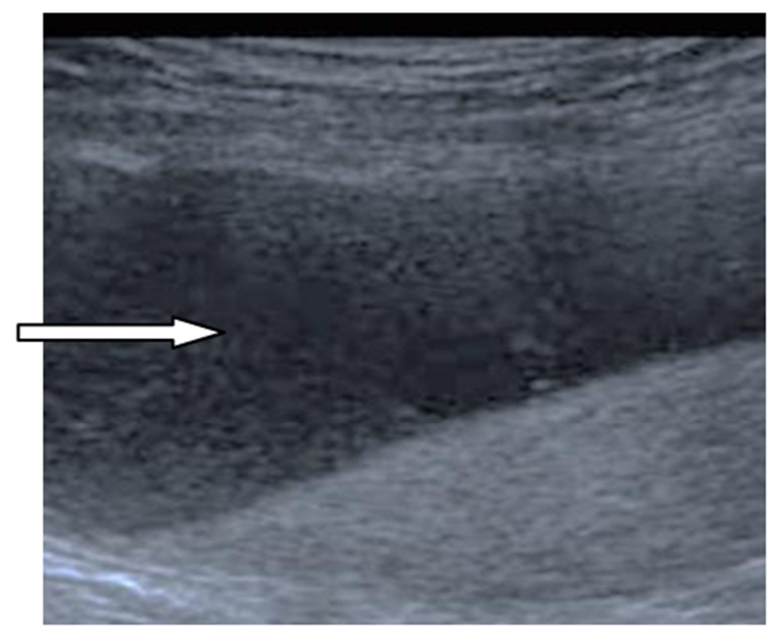

Figure 4. Male patient 45 years old presented with midline abdominal swelling after 5days from herinotomy operation. Transverse superficial sonography, demonstrate midline subcutaneous anechoic collection of fluid of anterior abdominal wall (seroma).

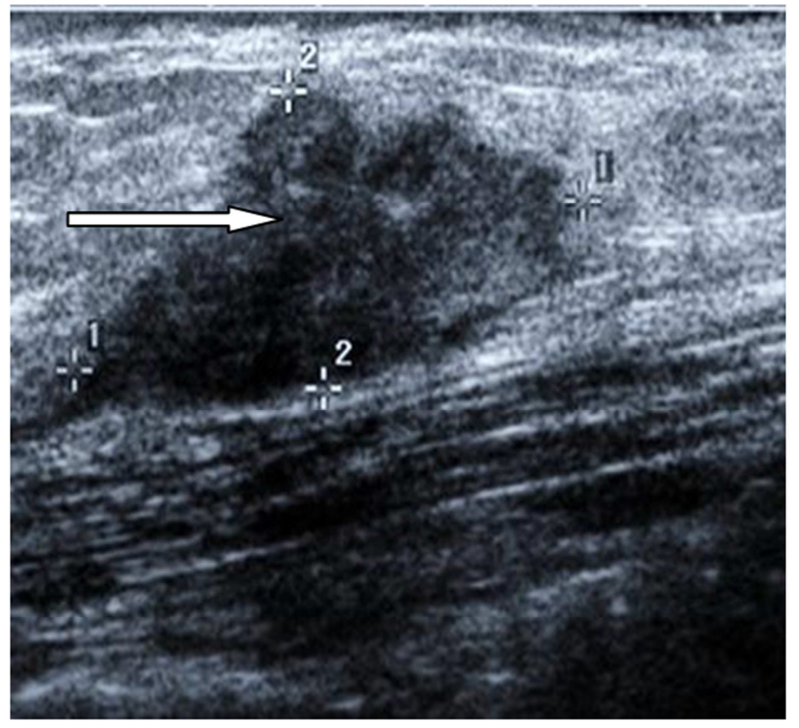

(A)

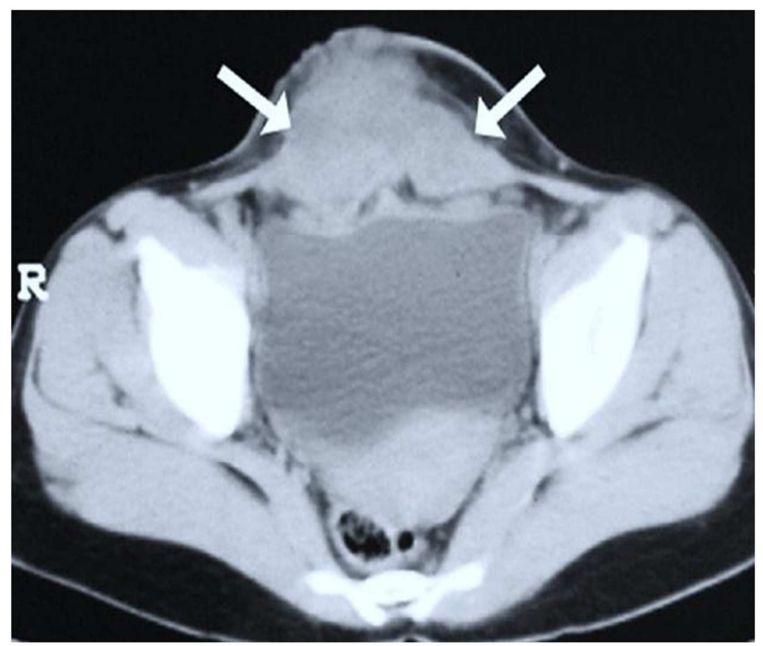

(B)

Figure 5. 40-year-old woman was presented with a 5-month history of pain during her menstruation at her abdominal scar from a caesarian section five years earlier. Fig.5 (A): Transverse superficial sonography, demonstrate well defined subcutaneous hypoechoic solid mass at site of scar of caesarean section measuring about $2.5 \times 1.5 \mathrm{~cm}$ (endometriosis). Fig.5(B):Axial CT abdomen, demonstrate hyperdense heterogeneous soft tissue density mass in site of scar of caesarean section measuring about $2.5 \times 1.5 \mathrm{~cm}$ (endometriosis).

\section{References}

[1] Gokhale S.: Sonography in identification of abdominal wall lesions presenting as palpable masses. J Ultrasound Med 2006; 25:1199-1209.

[2] Suleiman S, Johnston DE. The abdominal wall: an over looked source of pain. Am Fam Physician 2001; 64:431-438

[3] Diego A. A., Agnes C. S., Giovanna C., Claude B. S.. Abdominal Wall Hernias: Imaging Features, Complications, and Diagnostic Pitfalls at Multi-Detector Row CT1. Radio Graphics 2005; 25:6, 1501-1520 
[4] Rodriguez-Hermosa JI, Codina-Cazador A, Ruiz-Feliu B, Roig-Garcia J, Albiol-Quer M, Planellas-Gine P. Incarcerated umbilical hernia in a super-super-obese patient. Obes Surg 2008;18(7):893-5

[5] Halm JA, Heisterkamp J, Veen HF, Weidema WF. Long-term follow-up umbilical hernia repair: are there risk factors for recurrence after simple and mesh repair. Hernia 2005;9(4):334-7

[6] Bailey \& Love's short practice of surgery. Incisional hernia, 24th ed., 2004 By Arnold, 1260, wound healing 28.

[7] Louring-Andersen M, Hjorne FP, Skovdal J, Bisgaard T. Diagnosis and treatment of Spigelian hernia. UgeskrLaeger 2009;171(48): 3518-22

[8] Moles Morenilla L, Docobo Durántez F, Mena Robles J, Quinta Frutos R. Spigelian hernia in Spain. An analysis of 162 cases. Rev Esp Enferm Dig 2005; 97(5): 338-47.

[9] Neblett WW 3rd, Pietsch JB, Holcomb GW Jr. Acute abdominal conditions in children and adolescents. Surg Clin N Amer 1992;68:415-430
[10] Mura A, Kaneko H, Shirakura T, Shiba T, Imamura M. A case of primary abscess of the abdominal wall. J Jpn Surg Assoc. 2002; 63:728-31. Japanese.)

[11] Lefevre JH, Parc Y, Kernis S, Goasguen N, Benis M, Parc R, Tiret E. Risk factors for development of desmoids tumors in familial adenomatous polyposis. Br J Surg 2008;95:1136-9.

[12] Women DOUGLAS C., ROTIMI O., Extragenital endometriosis - a clinic pathological review of a Glasgow hospital experience with case illustrations, J Obstet Gynaecol, 2004, 24(7):804-808.

[13] Gandolfo N, Pretolesi F, Martinoli C, Crespi G, Derchi L. Radiol Med. Ju-Aug; 2002, 104(1-2):44-51.

[14] Stabile Ianora AA, Midiri M, Vinci R, Rotondo A, Angelelli G Abdominal wall hernias: imaging with spiral CT. Eur Radiol 2000; 10:914-919. 